\title{
WORLD SIMCHALLENGE — NEW SIMULATION IDEA FOR STUDENTS AND MEDICAL TEACHERS
}

\author{
Michael Czekajlo ${ }^{1,2}$, Marek Dabrowski ${ }^{1,3}$ \\ ${ }^{1}$ Polish Society of Medical Simulation, Poland \\ ${ }^{2}$ Hunter Holmes McGuire VA Medical Center, Department of Surgery, Richmond, United States of America \\ ${ }^{3}$ Department of Rescue and Disaster Medicine, Poznan University of Medical Sciences, Poznan, Poland
}

Disaster Emerg Med J 2017; 2(3): 137-139

Dear Editor,

Medical education is rapidly changing, with simulation becoming an integral part of these changes because of its ability to teach and objectively assess skills, critical thinking and management. Furthermore, simulation is a tool that is becoming integral in improving patient safety [1].

The American Association of Medical Colleges issued the following statement: "Simulation has the potential to revolutionize health care and address the patient safety issues if appropriately utilized and integrated into the educational and organizational improvement process" [2].

The International SimChallenge was established to provide medical students with a platform to use their knowledge in a friendly competition where they have to make decisions and realize the consequences. A report by the Carnegie Foundation entitled "Toward a Vision for the Future of Medical Education" criticized the current medical education system. Some of the criticisms were that: "medical training is inflexible, excessively long and not learner centered; There are poor connections between formal knowledge and experiential learning; and medical education does not adequately make use of the learning sciences" [3].

Medical student teams compete against each other by managing simulated critically ill patients. The teams are evaluated on the basis of their communication, teamwork, decision-making, situational awareness, task management and outcomes. It is an opportunity for students to use modern technology and learning science in the form of simulation in order to put into context all the information they are learning in medical school (Fig. 1, 2).

SimChallenge began as a local competition at Poznan University of Medical Sciences in 2011 to commemorate the success of the first academic year of its simulation program. In the years leading up to the development of the multidisciplinary simulation centre in Poznan the organizing committee had

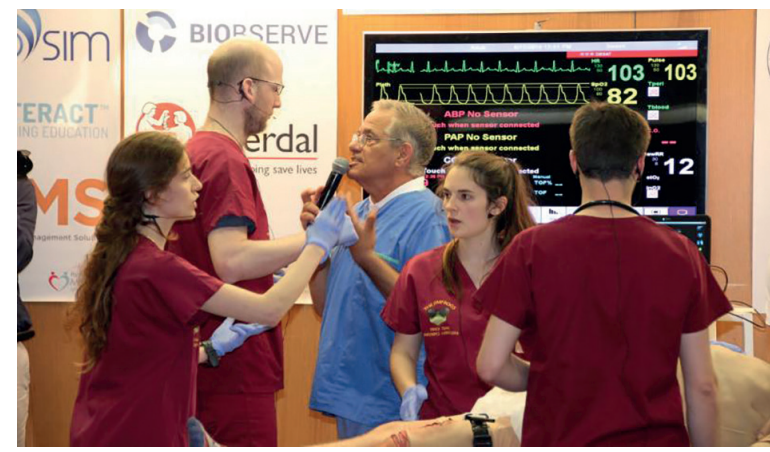

FIGURE 1. SimChallenge — team during scenario

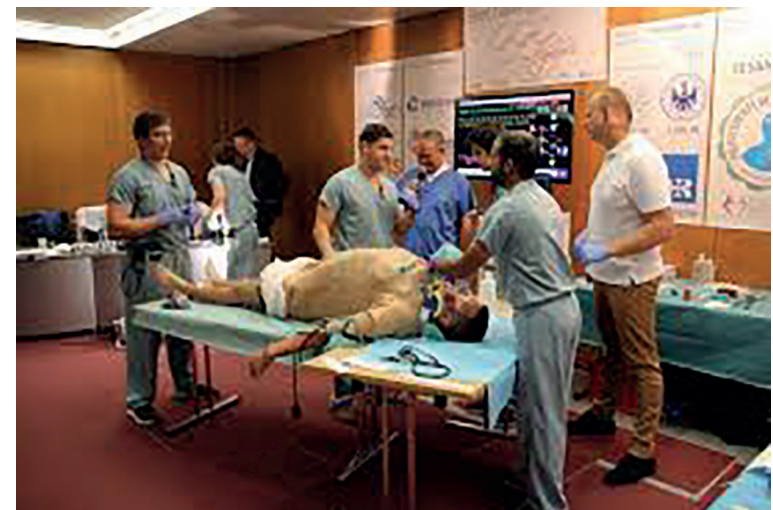

FIGURE 2. Team during final

Marek Dabrowski, Polish Society of Medical Simulation; Department of Rescue and Disaster Medicine, Poznan University of Medical Sciences, Poznan, Poland, e-mail: maro.dabrowski@gmail.com 
spent several years attending both the IMSH and SESAM simulation conferences.

The annual IMSH meeting had been hosting a SimWars competition that used simulated medical cases to test the knowledge and skills of medical teams. However, team members were composed of experienced healthcare providers. SimWars had also expanded into the annual conferences of the American College of Emergency Physicians and Society of Academic Emergency Medicine for Emergency Medicine Residency teams to compete [4].

The Polish SimChallenge organizers saw an opportunity to adopt the concept of SimWars for medical student education. In fact, during its first year the competition had been called SimWars but due to copyright of the SimWars competition at IMSH, the name was changed to SimOlympics and, again for copyright reasons, changed to SimChallenge.

In 2011, at the first SimChallenge competition in Poznan the event surpassed all expectations. The event alleviated any doubts by the Poznan medical faculty of the usefulness of simulation and motivated the students to want more simulation in the curriculum. The SimChallenge has proven not only to be a competition for medical students to showcase their abilities but also an event that demonstrates how well the faculty has prepared its students.

The SimChallenge has been an annual event at Poznan University of Medical Sciences since 2011 and in 2012 it became an annual event in Poland. Indeed, the year 2017 marks the 5th anniversary of the $\mathrm{Na}$ tional SimChallenge in Poland. The experience gained in organizing the competition has greatly improved the quality of simulation education in Poland. This experience has also translated to the international stage.

Poland had the honour of hosting the 20th anniversary meeting of SESAM in Poznan in 2014. During the SESAM congress, the first International SimChallenge of medical students took place (Fig. 3). The success of this first competition is best illustrated by the fact that the 4th SimChallenge is being hosted at the annual SESAM meeting in Paris, France.

The competition has had 8-10 teams compete in a tournament style bracket with two teams receiving the same case and the winning team moving on to the next round until 2 teams are left to compete in the Finals. The teams are debriefed by a panel of 3-4 judges. The popularity of the competition has required the organizers to increase the level of complexity of the final scenario due to the preparation of the students from year to year.

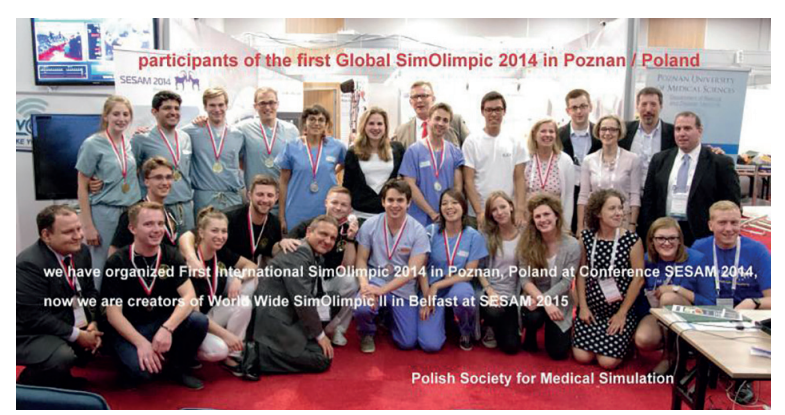

FIGURE 3. Organisers and participants - First International Finals of SimChallenge in Poznan 2014

All the cases during the competition present students with acute medical cases. Cases have included sepsis, trauma, pancreatitis, COPD exacerbation and a pregnant trauma patient, to name a few. In last year's competition in Lisbon, Portugal, the final round used a standardized patient wearing a surgical cutsuit which is an exoskeleton that allows the students to perform chest tube placement and cricothyrotomy to a patient who is awake, moving and screaming. One of the results of the SimChallenge has been demonstrating to faculty members that their students are capable of managing complex medical and surgical problems early in their career but require practice, repetition, feedback and a framework for translating the knowledge required of them into a critical thinking model that allows them to process all the patient data and make clinical decisions based on that data.

Simulation and the SimChallenge are tools that allows the students to gain experience and put them on the path to habituation. In addition to a being a fun way to learn, the SimChallenge allows them to compare their knowledge and skill levels with colleagues from different countries. We, the organizers of SimChallenge, have learned a great deal about medical education and our biases as to what students can manage along their educational career. Simulation is a tool that can help us objectively teach and assess students at an individual level meeting the goals of the Carnegie Foundation Report.

The International SimChallenge has been evolving over the past 4 years as we have learned from those who we are charged with teaching. The process has been enlightening and the future will undoubtedly lead to improvements in the way we teach the next generation of healthcare providers.

$$
\begin{array}{r}
\text { Michael Czekajlo } \\
\text { President of the Polish Society of Medical Simulation } \\
\text { Marek Dabrowski } \\
\text { Vice-president of the Polish Society of Medical Simulation }
\end{array}
$$


Conflict of interest: None declared.

\section{REFERENCES}

1. McGaghie WC, Issenberg SB, Cohen ER, et al. Does simulation-based medical education with deliberate practice yield better results than traditional clinical education? A meta-analytic comparative review of the evidence. Acad Med. 2011; 86(6): 706-711, doi: 10.1097/ ACM.0b013e318217e119, indexed in Pubmed: 21512370.
2. Medical Simulation in Medical Education: Results of an AAMC Survey. AAMC Report 2011.

3. Educating Physicians: A Call for Reform of Medical Schools and Residency. Carnegie Foundation Report. June 2010.

4. Okuda Y, Godwin SA, Jacobson L, et al. SimWars. J Emerg Med. 2014; 47(5): 586-593, doi: 10.1016/j.jemermed.2014.06.058, indexed in Pubmed: 25224173. 